\title{
Recurrent hypothenar hammer syndrome: An unusual presentation
}

\author{
Michael C-Y Ling BSc ${ }^{1}$, Bert Van Brenk MD FRCSC ${ }^{2}$, Jeffrey Howard $\mathrm{PhD}^{3,4}$, \\ Bing Siang Gan MD PhD FRCSC ${ }^{3,4}$ \\ ${ }^{1}$ University of Western Ontario, London; ${ }^{2}$ Peterborough Regional Health Centre, Peterborough; ${ }^{3}$ Hand \\ and Upper Limb Centre and ${ }^{4}$ London Wound Healing Group, St Joseph's Health Care, University of \\ Western Ontario, London, Ontario
}

\begin{abstract}
MC-Y Ling, B Van Brenk, J Howard, BS Gan. Recurrent hypothenar hammer syndrome: An unusual presentation. Can J Plast Surg 2001;9(5):199-203.

Upper extremity ischemia is a relatively rare phenomenon. A case of recurrent, surgically correctable, acute digital ischemia in a worker in the maple syrup industry is presented. The present case illustrates the importance of recognizing occupational exposure to risk factors. A review of the differential diagnosis, clinical work-up and literature surrounding acute vascular occlusion of the hand and upper extremity is presented.
\end{abstract}

Key Words: Acute digital ischemia; Hypothenar hammer syndrome

\section{Récurrence du syndrome hypothénarien du marteau : Tableau inhabituel}

RÉSUMÉ : L’ischémie des membres supérieurs est un phénomène relativement rare. On présente ici un cas de récurrence d’ischémie digitale aiguë justiciable d'un traitement chirurgical chez un travailleur de l'acériculture. Ce cas illustre l'importance de reconnaître l'exposition professionnelle à certains facteurs de risque. On passe en revue le diagnostic différentiel, les analyses cliniques et la littérature relatifs à l'occlusion vasculaire aiguë de la main et du membre supérieur.

A rterial insufficiency of the hand and upper limb is less common than that of the lower limb. The main differential diagnosis of acute ischemia of the hand and fingers includes arterial embolic events, thrombosis of the peripheral arterial system and vasospastic disorders. A case that illustrates the importance of the recognition of vocational exposure to risk factors and the possible contribution of systemic illness to the symptom complex of acute digital ischemia, thus aiding in narrowing the differential diagnosis is presented. This recognition allows for implementation of the appropriate diagnostic tests and facilitates the preoperative decision making process with respect to surgical intervention.

\section{CASE PRESENTATION}

A 49-year-old right-handed man presented to the local emergency department with the sudden onset of pain, pallor and dysesthesia of the tips of his left ring and small fingers. He described an intermittent sensation of cold associated with numbness and tingling, and a pale-bluish discoloration in the affected fingers. He denied ever having had these symptoms before. The symptoms started shortly after a long shift carrying heavy buckets of maple syrup out in the cold. This activity involved pouring the syrup by twisting the bucket handle and resulted in repeated pressure on the palm of the hand. In addition to the symptoms in the digits, the patient complained of pain in the palm that radiated to the wrist and elbow, and was particularly severe over the hypothenar eminence. He denied any recent injury to his fingers or hands and stated that he was otherwise healthy. Specifically, there was no previous cardiac history such as palpitations or previous myocardial infarction. No past history of joint or skin symptoms could be elicited. The patient was a nonsmoker, and his medications included acetylsalicylic acid (ASA), antibiotics for cellulitis of the leg, and acetaminophen. The patient was allergic to penicillin.

On examination, the range of motion and strength of the

Correspondence: Dr Bing Siang Gan, Hand and Upper Limb Centre, St Joseph's Health Care, 268 Grosvenor Street, London, Ontario

N6A 4L6. Telephone 519-646-6097, fax 519-646-6049, e-mail bsgan@lri.sjhc.london.on.ca 


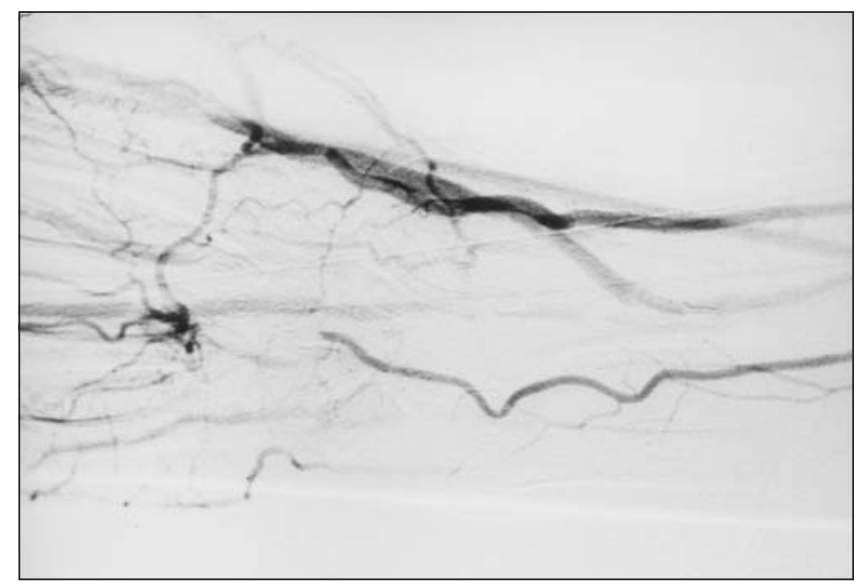

Figure 1) Digital subtraction angiogram showing an abrupt filling defect in the ulnar artery with a distal palmar arch filled from the radial artery

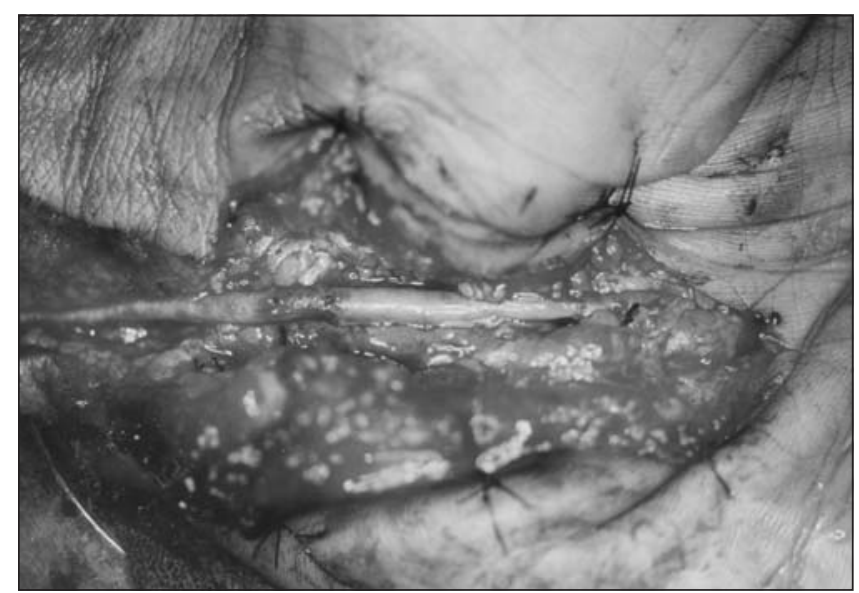

Figure 3) Resection and construction of the ulnar artery with a reversed vein segment obtained from the basilic vein. Microvascular anastomosis was performed using interrupted 9.0 nylon sutures

forearm musculature were normal, and no abnormal swelling or deformity was noted. The patient's ring and small fingers were pale and cold, with markedly diminished capillary refill. Turgor of the finger pads was normal. There was point tenderness over the hypothenar eminence in the region of the hook of hamate. Radial, ulnar, brachial and axillary pulses were palpable; however, the Allen's test was positive. There was a negative Tinel's sign at the cubital tunnel, and sensation in the distribution of the dorsal sensory branch of the ulnar nerve was normal. Two-point discrimination in the involved digits was elevated to greater than $10 \mathrm{~mm}$. Chest examination showed good respiratory sounds and the cardiac rhythm was regular without murmurs. A normal Allen's test was assessed on the contralateral hand. A pulse oximeter was able to detect a pulse with an oxygen saturation of greater than $95 \%$ in all digits, including the affected digits.

On the basis of the patient's history and physical examination, a provisional diagnosis of ulnar artery thrombosis was

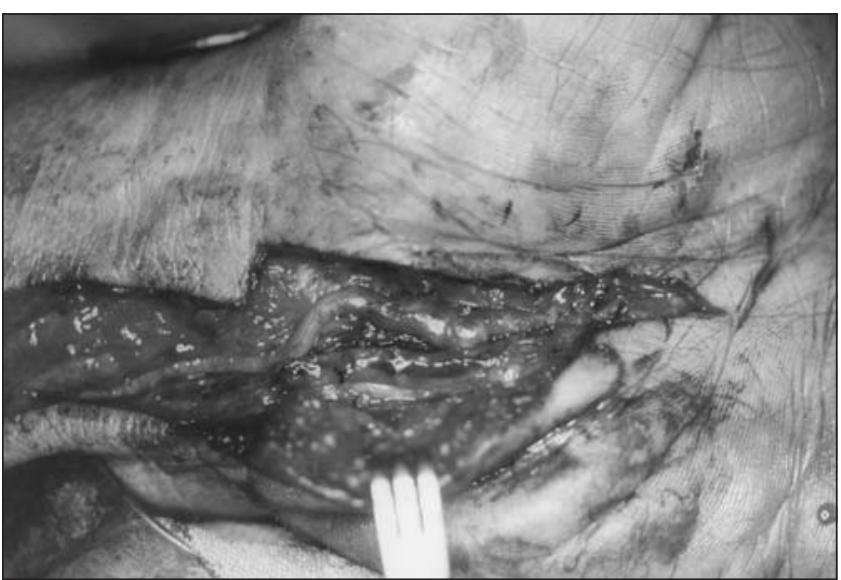

Figure 2) Surgical exploration of the wrist and Guyon's canal revealed a tortuous and thrombosed ulnar artery segment

made and additional tests performed included an arterial Doppler examination. The Doppler test results showed compromised blood flow to the ulnar three digits that only occurred during radial artery compression, thus suggesting an abnormal flow through the ulnar artery, with a complete palmar arch perfusing the ulnar digits through the radial artery. Flow assessment indicated normal digital finger pressures and waveforms in all digits. Critical ischemia was thought not to be present; however, the symptoms persisted in addition to severe cold intolerance, confining the patient to his home.

Despite the relatively normal Doppler test, an angiogram was performed to rule out definitively the presence of ulnar artery thrombosis, and this test confirmed the presence of total segmental occlusion of the ulnar artery proximal to Guyon's canal (Figure 1). The patient was taken to the operating room for exploration of the ulnar artery. The content of Guyon's canal revealed a considerable amount of inflammation. The ulnar artery at this region was tortuous and completely thrombosed (Figure 2). The ulnar nerve within Guyon's canal was significantly inflamed and hyperemic. Following excision of the thrombosed segment of the ulnar artery and release of the tourniquet, little back-flow from the distal superficial palmar arch was noted. Therefore, a reversed segment of the ipsilateral basilic vein was used to reconstruct the resected ulnar artery (Figure 3). After release of the clamps, good flow was observed through the graft.

Pathological examination of the resected left ulnar artery segment showed a $4.3 \mathrm{~cm}$ tubular structure that possessed a luminal thrombus with recent blood clot formation. Signs of underlying atherosclerosis were also noted. The outer third of the media and the adventitia were infiltrated with inflammatory cells and fibroblasts (Figure 4). These signs of chronic inflammation were not consistent with an acute traumatic event with secondary thrombosis, but were considered to be more suggestive of a thrombotic event on a chronically inflamed vessel wall, possibly related to previous repetitive trauma. 
Postoperatively, the patient reported numbness of the ring and small fingers, but physical examination showed a normal two-point discrimination. The symptoms of cold intolerance improved markedly. Follow-up Doppler examination six weeks postoperatively showed normal perfusion of the ulnar digits, even on compression of the radial artery, thus confirming a patent graft and improved digital flow. The patient was advised to take $325 \mathrm{mg}$ of ASA daily for three months and then to discontinue this medication. The subjective dysesthesia disappeared over the course of three months. The fingers remained warm and had improved capillary refill.

Interestingly, approximately two years later, the patient presented again with similar symptoms and physical examination. Doppler tests again confirmed occlusion of the ulnar artery. Operative exploration of Guyon's canal was again undertaken. An arteriotomy was performed just proximal to the previous vein graft segment, and a Fogarty catheter was used to perform a thrombectomy of the fresh clot. The patient was started on a heparin drip and subsequently discharged on long term ASA therapy. The patient was advised to avoid trauma to his hands in his vocational and avocational activities. One year following the second surgery, the patient was symptom free.

\section{DISCUSSION}

Arterial insufficiency of the hand and upper limb is less common than that of the lower limb (1). The differential diagnosis of upper extremity and digital ischemia should include arterial embolic events, thrombosis of the peripheral arterial system and vasospastic disorders $(1,2)$. Specific risk factors and subtle differences in the presentation and physical examination of each of these causes of digital ischemia need to be identified to narrow the differential diagnosis.

Arterial embolic events present typically with the sudden onset of pain followed by Raynaud's phenomenon, paresthesias and diminished pulses. The majority of the emboli, representing $70 \%$ of all emboli, are from a cardiac source (1). In individuals with these emboli, a history of valve replacement, atrial fibrillation and/or myocardial infarction may be noted. An electrocardiogram may aid in the diagnosis of emboli with a cardiac origin. A smaller proportion of emboli are secondary to subclavian artery compression.

Vasospastic disorders encompass a spectrum of conditions in which no history of trauma or risk factors of embolic disease are reported. These conditions involve widespread disease with systemic symptoms, and include conditions such as Buerger's disease and primary Raynaud's disease. Smokers are at a higher risk for these conditions, which present with claudication, rest pain or ulcerations.

A thorough head-to-toe physical examination, focusing on signs of systemic disease, collagen vascular abnormalities and pre-existing illnesses, further aids in identifying the underlying etiology. Routine examination of the involved hand includes inspection for colour, temperature and morphology. Peripheral pulses and capillary refill time can be compared with the contralateral side. Physical examination may reveal subcutaneous thickening and tenderness over the areas of

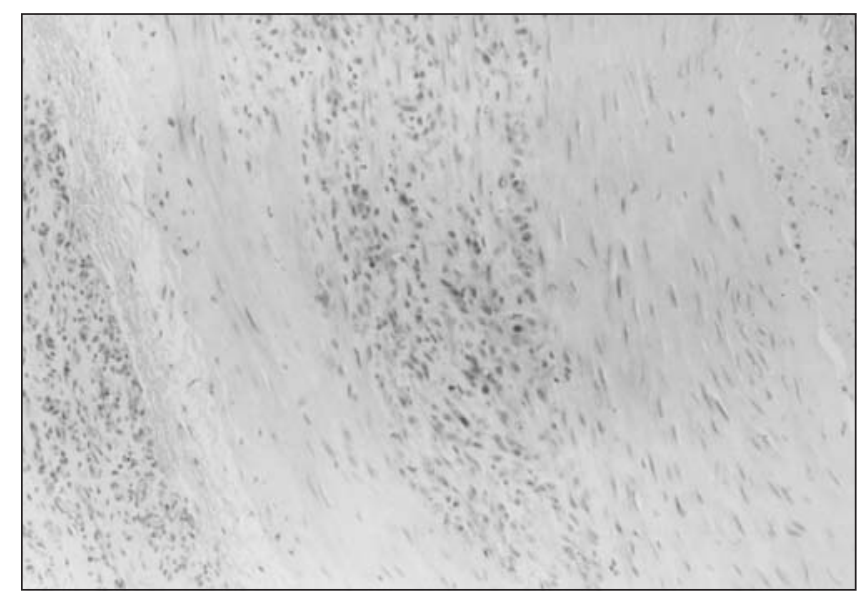

Figure 4) Histological cross section of the resected ulnar artery segment showing a luminal thrombus (right side) with atherosclerosis of the vessel wall and infiltration of fibroblasts and chronic inflammatory cells in the outer third of the media (left side)

thrombosed arteries, including around the hypothenar eminence. In the presence of an aneurysm, a mass may be palpated. The Allen's test, which is of particular importance in testing for radial and ulnar artery contribution to the superficial arch, as well as for the patency of the arch, can give an indication of the patency of the major arteries supplying the hand.

The flow dynamics to the hand can be further delineated by diagnostic modalities such as blood flow Doppler mapping, impedance plethysmography or angiography. Doppler mapping is a proven, noninvasive technique of visualizing vascular anatomy (3) that plays an important role in preoperative assessment and postoperative follow-up. Being noninvasive, it has the advantage of not affecting vascular tone, thus giving reliable results. Doppler mapping characterizes flow graphically and numerically, which allows for longitudinal quantitative comparison over time to assess deterioiration or improvement in blood flow patterns.

Angiography plays a key role in the diagnosis and plan for treatment. It serves to rule out an embolic event, to confirm the exact location of vascular damage, to distinguish between thrombus and aneurysm, and to investigate embolic events in the digital arteries $(1,2)$. Features on angiography may include irregularity in filling, corkscrew configuration (4), occlusion, and normal proximal and collateral arteries $(2,4,5)$. However, the use of contrast material may induce or aggravate vasospasm, thus resulting in a misdiagnosis.

\section{HYPOTHENAR HAMMER SYNDROME}

As illustrated in the present case, the recognition of occupational exposure to risk factors, in conjunction with signs of acute digital ischemia, is important in formulating a differential diagnosis and an approach to management. Hypothenar hammer syndrome (HHS) is the name used to describe the signs and symptoms associated with hand and finger ischemia resulting from trauma and thrombosis of the ulnar artery. It most often develops after repeated trauma to the hypothe- 
nar eminence; however, a single traumatic event that causes HHS has also been reported (2). HHS usually presents on the dominant hand, with the ring and little fingers being the most commonly affected digits. It is more common in men, and in $75 \%$ of cases, it has only unilateral involvement $(2,5)$. HHS was first described in 1934 by Von Rosen (6). As the name suggests, the syndrome develops when the hypothenar eminence of the hand is used as a hammer in occupational or recreational activities. Conn et al (7) reported the case of a 23-year-old factory worker who acutely struck the palm of his right hand against a metal bar while attempting to loosen a screw. The patient subsequently developed pain, swelling and ecchymosis over his hypothenar eminence. Two weeks later, he developed ischemic pain in his right hand. On surgical exploration, the ulnar artery was discovered to be thrombosed over the hamate. The thrombosed segment was resected, and the patient did well postoperatively (7).

The pathogenesis of HHS requires an understanding of the anatomy of the hand. The ulnar artery is the major artery of the hand, contributing $75 \%$ of the blood flow to the superficial palmar arch. While in the well protected Guyon's canal, the ulnar artery divides into the superficial and deep branches. The superficial ulnar artery leaves Guyon's canal and travels a distance of approximately 2 to $3 \mathrm{~cm}$ before becoming the superficial palmar arch. In the short distance distal to Guyon's canal, the artery lies directly on the hook of the hamate bone. On its palmar side, the artery is only protected by the palmaris brevis muscle, the superficial aponeurosis, subcutaneous tissue and skin. It is here that the ulnar artery is most vulnerable to extrinsic forces, such as pushing, squeezing and hammering, that compress it against the hamate bone, which acts as an anvil $(2,8)$.

Repetitive and chronic trauma to the ulnar artery may have several outcomes, including vasospasm, aneurysm formation or occlusion. Compression of the ulnar artery may result in damage to the intima, thus exposing the subendothelial collagen to platelets and subsequent thrombus formation. Subsequently, embolic events may result in acute occlusion of digital arteries (2). If the damage extends beyond the intima to involve the media, aneurysm of the ulnar artery ensues. Another response to chronic trauma, is thickening and scarring of the periadventitial tissue, with resultant extrinsic compression and occlusion of the ulnar artery $(1,2)$. The most common form of trauma is occupational in nature, particularly in individuals who use high speed vibrating tools or perform activities that apply repetitive forces on the hypothenar eminence $(2,8,9)$. In many cases, the trauma that produces arterial occlusion is trivial and ignored by the patient, thus making diagnosis of the condition difficult.

HHS is a reversible and treatable cause of digital ischemia and it is, therefore, important to make an early diagnosis. Therapeutic options for HHS vary from symptomatic treatment, to sympathetic blockade, to surgical resection with or without reconstruction (2). All patients with HHS can be advised to avoid aggravating factors such as cold temperatures and smoking. Identifying and eliminating traumatic activities from one's lifestyle slows the progression of the underlying pathology.

HHS falls into the category of thrombosis of the peripheral arterial tree, for which a history of chronic occupational injury is often present but incidental to the patient. Patients with this type of history usually have symptoms of cold hypersensitivity, dysesthesias, finger pain, claudication and pain over the hypothenar eminence (2). The most common sign of HHS is Raynaud's phenomenon, in which there is coldness of the fingers with phasic colour changes precipitated or aggravated by cold temperatures $(2,9)$.

Thrombosis triggers a hypersympathetic reflex response, resulting in vasospasm and further compromise of the arterial blood supply to the digits $(1,2)$. Therefore, therapy has been aimed at blocking the sympathetic drive with stellate ganglion blockade or removing the local sympathetic centre with a dorsal sympathectomy (2). Alternatively, removal of the sympathetic triggering stimulus, via fibrinolysis or surgery, is possible. Indication for fibrinolysis includes the presence of multiple peripheral emboli that precludes surgery, but if left untreated may result in permanent changes (2). Surgical resection of the thrombosed segment of the ulnar artery without reconstruction removes the cause of vasospasm and eliminates the risk of embolic events.

The decision to reconstruct the resected segment is dependent on the adequacy of digital flow that exists following resection. Doppler flow, in addition to measuring digital pressures, is used to determine whether the resection of the segment is adequate in preserving digital flow, or whether supplementation of the flow with end-to-end anastomoses or vein graft reconstruction is required. In a case described by Zimmerman (1), in which intraoperative plethysmography was used to assess digital blood flow, a Digital Brachial Index value of less than $70 \%$ was set as the critical value in determining the necessity of reconstructing the ulnar artery. Reconstruction options include end-to-end anastomosis following resection or vein graft interposition.

Assurance of a patent reconstruction begins intraoperatively at which time resection of the thrombosed segment is taken back to normal intima. The normal intima needs to be reapproximated with normal intima of vein graft in a tension-free repair $(1,2)$. Once satisfactory reconstruction has been achieved, good pulsatile flow through the graft can be observed. Postoperatively, intravenous heparin over a short term period or long term ASA may be implemented to minimize the risk of thrombosis recurrence.

\section{CONCLUSION}

The present case illustrates the importance of a thorough history and physical examination. It provides a framework to approach the diagnosis and treatment of a surgically correctable disorder causing ischemia of the upper extremity, a phenomenon that is relatively rare.

\section{REFERENCES}

1. Zimmerman NB. Occlusive vascular disorders of the upper extremity. Hand Clin 1993;9:139-50. 
2. Van de Walle PM, Moll FL, De Smet AA. The hypothenar hammer syndrome: update and literature review. Acta Chir Belg 1998;98:116-9.

3. DiBenedetto MR, Nappi JF, Ruff ME, Lubbers LM. Doppler mapping in hypothenar syndrome: an alternative to angiography. J Hand Surg [Br] 1989;14:244-7.

4. Hammond DC, Matloub HS, Yousif NJ, Sanger JR. The corkscrew sign in hypothenar hammer syndrome. J Hand Surg [Br] 1993;18:767-9.

5. Kreitner KF, Duber C, Muller LP, Degreif J. Hypothenar hammer syndrome caused by recreational sports activities and muscle anomaly in the wrist. Cardiovasc Intervent Radiol 1996;19:356-9.
6. Von Rosen S. Ein Fall von Thrombose in der Arteria ulnaris nach Einwirkung von stumpier Gewalt. Acta Chir Scand 1934;73:500

7. Conn J Jr, Bergan JJ, Bell JL. Hypothenar hammer syndrome: posttraumatic digital ischemia. Surgery 1970;68:1122-8.

8. Duncan WC. Hypothenar hammer syndrome: an uncommon cause of digital ischemia. J Am Acad Dermatol 1996;34:880-3.

9. Pineda CJ, Weisman MH, Bookstein JJ, Saltzstein SL. Hypothenar hammer syndrome: Form of reversible Raynaud's phenomenon. Am J Med 1985;79:561-70. 